\title{
Typical CT and MRI signs of hepatic epithelioid hemangioendothelioma
}

\author{
LU GAN, RUIPING CHANG, HUALAN JIN and LI YANG \\ Department of Radiology, Chinese People's Liberation Army General Hospital, Beijing 100853, P.R. China
}

Received January 9, 2015; Accepted January 12, 2016

DOI: $10.3892 / 01.2016 .4149$

\begin{abstract}
To investigate the typical magnetic resonance imaging (MRI) and computed tomography (CT) features of hepatic epithelioid hemangioendothelioma (HEH), the CT and MRI findings of 14 histopathologically confirmed cases of HEH were retrospectively analyzed. Non-contrast and dynamic contrast-enhanced scans were conducted in all cases. A total of 229 lesions were detected in the 14 cases. All cases were classified as one of three types: (i) Solitary nodular type ( 1 case, $7 \%$ ); (ii) multifocal nodular type (11 cases, $79 \%$ ); or (iii) diffuse type (2 cases, 14\%). The diameter of the lesions ranged from 5 to $105 \mathrm{~mm}$. For the first two types (solitary and multifocal nodular types), the CT findings included low density lesions with clear margins on non-contrast scans, centripetal enhancement in arterial phase, and homogeneous enhancement in the portal venous and delay phases. The findings of non-contrast MRI scans for these two types included low signal intensity on T1-weighted images, heterogeneous high signal intensity on T2-weighted images, and heterogeneous high signal intensity on diffusion-weighted images. The lesions were predominantly located in submarginal areas. On contrast-enhanced MRI, the findings for the first two types included peripheral ring-like enhancement with a central low signal intensity ('black target-like' sign) and a central enhanced core surrounded by a low signal intensity halo ('white target-like' sign). The findings for the third HEH type (diffuse type) on CT and MRI scans included low density or heterogeneous signal intensity lesions involving regions of part or the whole liver, coalescent lesions ('strip-like' sign), and gradual enhancement along central vessels ('lollipop' sign). Collectively, these findings indicate that the 'white target-like' sign, 'black target-like' sign, 'lollipop' sign and 'strip-like' sign, in addition to capsular contraction and submarginal location, on CT and MRI imaging may have implications for the
\end{abstract}

Correspondence to: Professor Li Yang, Department of Radiology, Chinese People's Liberation Army General Hospital, 28 Fuxing Road, Beijing 100853, P.R. China

E-mail: 13805366900@126.com

Key words: computed tomography, magnetic resonance imaging, hepatic epithelioid hemangioendothelioma diagnosis of HEH. Furthermore, a variety of MRI sequences may provide additional information for the differential diagnosis of HEH.

\section{Introduction}

Hepatic epithelioid hemangioendothelioma (HEH) is a rare vascular tumor of endothelial origin with low- to intermediate-grade malignancy (1). HEH has a prevalence of 1 per 100,000 population (2). HEH may present as a solitary liver nodule or, more frequently, as multifocal liver nodules. Previous studies have revealed that HEH tends to coalesce into diffuse lesions in the late stages of the disease (3). Conventional treatments of HEH include surgical resection or liver transplantation. Previously, certain novel systemic drugs have been used in the treatment of HEH, including thalidomide and sorafenib $(4,5)$. The prognosis for patients with HEH is considered much more favorable compared with that of other hepatic malignancies, with a 5-year survival rate of $43-55 \%$ (6).

Previously, HEH was frequently misdiagnosed as metastasis or, more rarely, as primary tumor of the liver, based on imaging and pathology (7). Pathological studies have indicated that myxoid, hyaloplasm and fiber compositions are present in the central part of $\mathrm{HEH}$, which determine the appearance of the computed tomography (CT) and magnetic resonance imaging (MRI) scans and the enhancement patterns of the tumor $(6,8)$. The peripheral region of the lesion, which is rich in tumor cells, demonstrates rim enhancement on the arterial phase, while the central part, which is rich in fiber composition, demonstrated delayed enhancement (9). Similar enhancement patterns have frequently been identified in cholangiocarcinoma (10). In addition, the fibrous contraction in the central region of the lesion caused the adjacent capsular contraction (3). The myxoid and hyaloplasm composition in the central region of HEH demonstrates no enhancement and the peripheral region demonstrates rim enhancement, termed the 'black target sign' or 'bulls eye sign', which may be frequently identified in metastatic tumors and hepatic abscesses (6).

Clinical presentation and history may be useful for the differential diagnosis of $\mathrm{HEH}$, metastatic tumors and abscesses. The peripheral regions of certain hemangiosarcomas and atypical hemangiomas often demonstrate progressive enhancement, while the central necrosis or cystic regions demonstrate no enhancement (11). Differentiating 
$\mathrm{HEH}$ from the aforementioned tumors is challenging. At present, with the development and popularity of CT and MRI techniques, increasing numbers of characteristics associated with HEH have been extracted from CT and MRI images, including the 'target sign' and 'lollipop sign' (12). In addition, apparent diffusion coefficient (ADC) maps may be useful in revealing the malignant potential of the tumor (13). The final diagnosis of $\mathrm{HEH}$ depends on the pathology.

Thus, it is important to increase awareness of the imaging characteristics of HEH. In the current study, the CT and MRI findings of 14 cases of histopathologically confirmed $\mathrm{HEH}$ were retrospectively evaluated.

\section{Materials and methods}

Patients. Data from 14 cases of HEH, treated between 2010 and 2014 in the Chinese People's Liberation Army General Hospital (Beijing, China), were retrospectively collected. Informed consent from the patients was not required for this retrospective study as patient privacy was maintained. The diagnoses of 2 cases were determined by surgery, and 12 cases were confirmed by needle biopsy, with hematoxylin and eosin and immunohistochemical staining (BenchMark; Ventana Medical Systems, Inc., Tucson, AZ, USA). The immunohistochemical assessment was positive for cluster of differentiation 34 (CD34) and factor VIII-related antigen (12). The male:female ratio was $1: 1$, and the mean age of the patients was 43.5 years (range, 24-70 years). The percentage of asymptomatic cases was $50 \%$ ( 7 cases), whilst $21 \%$ ( 3 cases) presented with right upper quadrant pain, $28.5 \%$ (4 cases) presented with weight loss, and $7.1 \%$ ( 1 case) presented with jaundice and $7.1 \%$ ( 1 case) with fever. Only 1 case had a history of lung cancer resection. In 1 case, the level of carcinoembryonic antigen (CEA) was increased slightly (6.14 $\mu \mathrm{g} / \mathrm{l}$; normal range, $0.1-5 \mu \mathrm{g} / \mathrm{l})$. The level of carbohydrate antigen 125 (CA125) was also increased in 1 case $(63 \mathrm{U} / \mathrm{ml}$; normal range: $<5$ units/ml).

Scan protocol. Non-contrast and two-phase dynamic contrast-enhancement CT scans were performed in 7 cases using a Siemens Sensation Cardiac 64 CT scanner (Siemens AG, Munich, Germany). In addition, 9 patients were examined by MRI (Signa Excite HD 1.5T; GE Healthcare Life Sciences, Shanghai, China). Patients were imaged in the supine position with a surface phased-array coil. For the complete evaluation of liver lesions, breath-hold transverse T2-weighted fast spin-echo sequences were initially performed, followed by transverse T1-weighted dual-echo in-phase and out-phase sequences, with a $5-\mathrm{mm}$ slice thickness and $1-\mathrm{mm}$ interspace. Three-dimensional fat-saturated T1-weighted dynamic contrast-enhanced sequences were performed during suspended respiration. Gadopentetate-dimeglumine (Gd-DTPA; $0.1 \mathrm{mmol} / \mathrm{kg}$; GE Healthcare Life Sciences) was injected intravenously at a rate of $2 \mathrm{ml} / \mathrm{sec}$ by a power injector. Dynamic contrast-enhanced MRI was performed in the transverse plane with a 3-mm slice thickness and no interspace at baseline (pre-contrast), followed by the hepatic arterial-dominant (20-25 sec), portal venous (30-35 sec) and delay (300-360 sec) phases after contrast injection. Before dynamic contrast-enhanced imaging, transverse respiratory-triggered diffusion-weighted single-shot echo-planar imaging sequence was performed with tri-directional diffusion gradients by using two b-values of 0 and $800 \mathrm{sec} / \mathrm{mm}^{2}$.

Image analysis. All images were analyzed separately by two radiologists. Two reviewers analyzed all images with regard to the following aspects: Number, location and size of lesions; morphological features; intensity; and characteristics of dynamic contrast-enhanced images. By consensus through a joint review of the recorded images, each case was finally classified as one of the following types: (i) Solitary nodular type, a solitary lesion that has a diameter of $<30 \mathrm{~mm}$; (ii) multifocal type, multifocal lesions that may be separated from one another; or (iii) diffuse type, coalescent multifocal or diffuse lesions that have no clear margins between each other.

\section{Results}

Solitary nodular type. In 1 case, a solitary, low-density nodular lesion with an irregular shape was observed on non-contrast CT imaging, with slightly progressive enhancement on contrast-enhanced imaging (Fig. 1).

\section{Multifocal nodular type}

Number, size and distribution of lesions. Multifocal lesions were detected in 11 cases: 46 lesions were detected by CT in 4 cases, and 178 lesions were detected by MRI in 7 cases. In 4 cases $(28.5 \%)$, the lesions were located in the right lobe of the liver, whilst in 1 case $(7 \%)$ lesions were in the left lobe, and 6 cases $(43 \%)$ involved the whole liver. The lesions were predominantly located in submarginal areas of the liver. Coalescent lesions were detected in a number of cases. The hepatic or portal veins were involved in 6 cases (42\%). The diameters of the lesions ranged from 5 to $105 \mathrm{~mm}$.

Patterns of lesion signal intensity. The lesions exhibited low density with clear margins on CT non-contrast imaging, and slight centripetal enhancement from the arterial to portal phases on contrast-enhanced CT imaging. On T1-weighted imaging (T1WI), the lesions exhibited low signal intensity. On T2-weighted imaging (T2WI), the lesions showed high signal intensity relative to the liver parenchyma, and the scattered or coalescent lesions, located in submarginal areas, showed a 'white target-like' sign or 'strip-like' sign; the two-layered 'target-like' appearance was formed by the high-signal intensity core and peripheral slightly hyperintense halo. On diffusion-weighted imaging (DWI), the lesions showed a high-signal intensity halo outside of the central slightly high-signal intensity core. 'Target-like' configurations were detected in 141 cases (60.5\%) (Figs. 2 and 3). On dynamic contrast-enhanced MRI scans, the enhancement features varied with different blood supply patterns. In total, 160 lesions (70\%) exhibited gradual peripheral ring-like enhancement patterns, with central low signal intensity in the arterial to portal venous and delay phases, and 51 cases (24\%) showed peripheral ring-like enhancement patterns with central low signal intensity in arterial phase, and the enhanced lesions were surrounded by a thin hypointense ring in the portal venous or delay phases ('black target-like' sign) (Fig. 3). Liver capsular flattening or retraction was observed in 60 lesions (27\%), and 1 case (4\%) exhibited 'strip-like' 


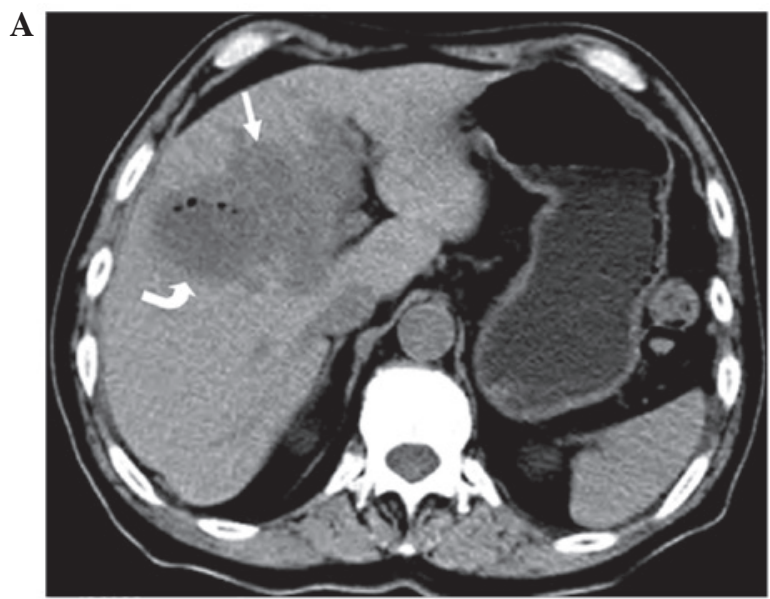

$\mathbf{B}$

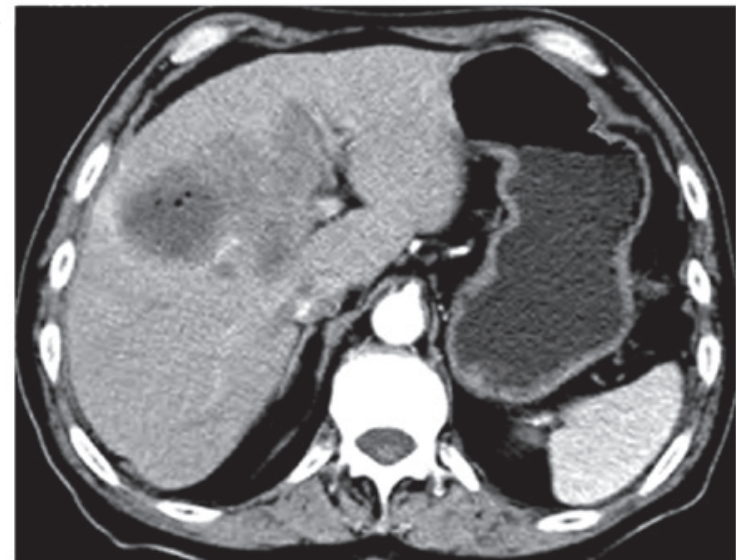

$\mathbf{C}$

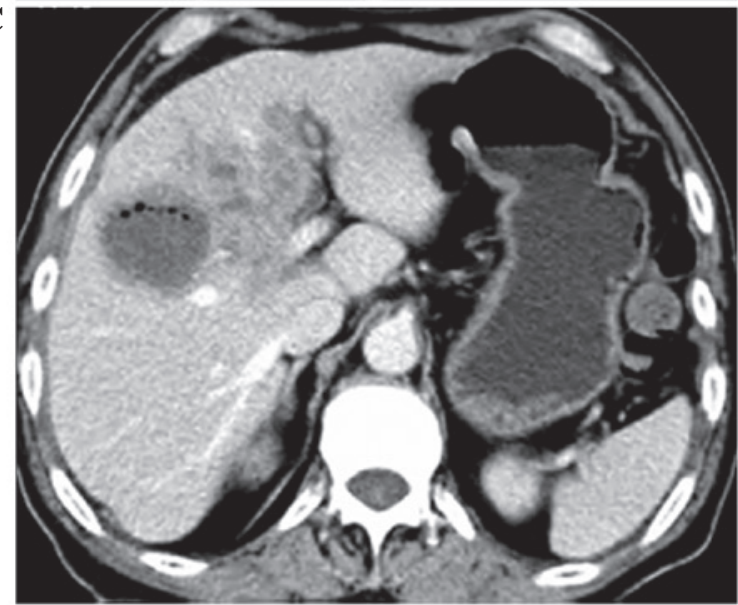

Figure 1. A 45-year-old male with solitary nodular-type HEH. (A) The HEH nodule (straight arrow) had a low-density appearance on non-contrast computed tomography imaging, and a liver abscess (curved arrow) with tiny air bubbles was found adjacent to the HEH lesion. (B) The HEH nodule showed slight enhancement in the arterial phase. (C) The HEH nodule showed gradual ring-like enhancement. $\mathrm{HEH}$, hepatic epithelioid hemangioendothelioma.

coalescence of multifocal nodules (Fig. 3). Nodular enhancement along the central vessels ('lollipop' sign) was observed in 10 lesions (4\%) (Fig. 4). Heterogeneous-intensity masses surrounded by several nodules were present in 5 cases (36\%); the masses exhibited peripheral nodular enhancement in the arterial phase and gradual homogeneous enhancement in portal venous phase. The transient abnormal perfusion of the peripheral liver parenchyma was visualized in the arterial phase and disappeared in portal venous phase (Fig. 4).
Diffuse type. Diffuse lesions throughout the whole liver were observed in 2 cases. The CT features included diffuse low-density lesions with minimal residual areas of normal liver parenchyma, in addition to nodular or irregular enhancement in the arterial phase and gradual enhancement in portal venous phase. The MRI features included heterogeneous low signal intensity on T1WI and high signal intensity on T2WI, with patchy uniform signal intensity within the lesions. Nodules and masses coexisted in the 2 cases. Nodules exhibited 'target-like' signs on non-contrast imaging and heterogeneous enhancement on contrast-enhanced imaging, while masses showed 'strip-like' enhancement along central vessels in the arterial phase and gradual enhancement in the portal venous and delay phases. Stenosis or occlusion of the portal and hepatic veins were also detected (Fig. 5).

Other findings. Metastatic tumors in the lung, spleen, thoracic vertebra, ilium or thoracic wall were detected in 4 cases (28.5\%), a liver abscess was observed in 1 case (7\%), and ascites was present in 1 case $(7 \%)$. The hepatic or portal veins were involved in 6 cases $(42 \%)$.

Methods of definitive diagnosis. All cases were misdiagnosed prior to surgery or needle biopsy: 2 cases were diagnosed as malignant tumor of the liver, 4 cases were diagnosed as cholangiocellular carcinoma, 4 cases were diagnosed as metastatic tumors, 1 case was diagnosed as hepatic fibrosis and 2 hypovascular cases were undetermined.

\section{Discussion}

The present study investigated the CT and MRI characteristics of HEH. The findings revealed a number of typical CT and MRI signs associated with $\mathrm{HEH}$, most notably the 'white target-like' sign on contrast-enhanced MRI.

$\mathrm{HEH}$ is a rare malignant tumor of vascular origin. It was described as being characterized by the presence of 'epithelioid' or 'histiocytoid' endothelial cells in 1982 by Weiss and Enzinger (14), who used the term 'epithelioid hemangioendothelioma' to designate these biologically 'borderline' neoplasms. HEH appears to have a clinical course between benign hemangioma and angiosarcoma. The World Health Organization classifies HEH as a malignant tumor (10). In the majority of patients, both lobes of the liver are involved, and lung, peritoneum, lymph nodes and bone are the most common sites of simultaneous extra-hepatic involvement $(1,15)$. HEH predominantly occurs in adult females; the mean age of patients is $\sim 41.7$ years, and the female:male ratio is 1.6-2.0:1. The etiology of HEH remains unknown; however, it may be associated with oral contraceptive use, exposure to polyethylene, trauma or viral hepatitis $(2,6,16)$.

In the current study, a total of 229 lesions were detected, most of which were located in the submarginal areas of the liver, and $56 \%$ of which had capsular flattening or retraction; this was lower than that reported by Miller et al (3) and Paolantonio et al (17), but similar to that reported by Zhao et al (18), in which capsular retraction was observed in $59.5 \%$ of HEH in Chinese patients. MRI has been demonstrated to have advantages over CT in the detection of submarginal and small size lesions. Certain studies indicated that submarginal 



Figure 2. A-58-year female with multifocal nodular-type HEH. (A) On T2-weighted imaging, HEH nodules (straight arrow) showed a two-layered 'target-like' appearance with central high signal intensity; the high-signal intensity core was surrounded by a peripheral slightly hyperintense halo. The lesions were predominantly located in submarginal areas of the liver (curved arrow). (B) On TI-weighted imaging, the lesions had a low-signal intensity appearance. (C) On contrast-enhanced T1-weighted fat-suppressed images, the lesions exhibited gradual ring-like enhancement (arrow). HEH, hepatic epithelioid hemangioendothelioma.

A



C

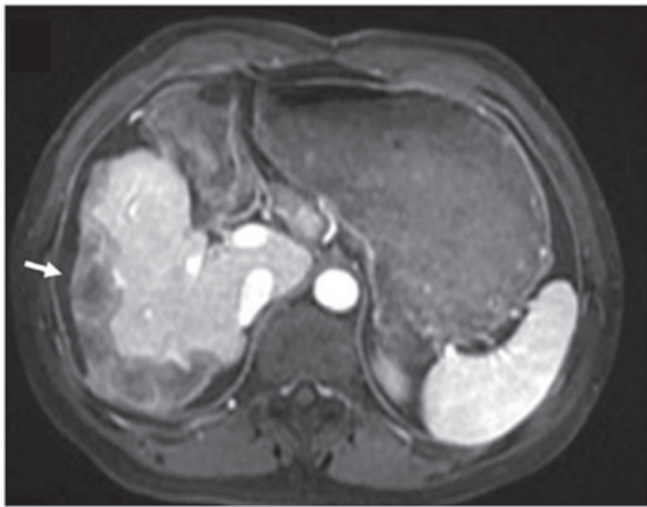

B

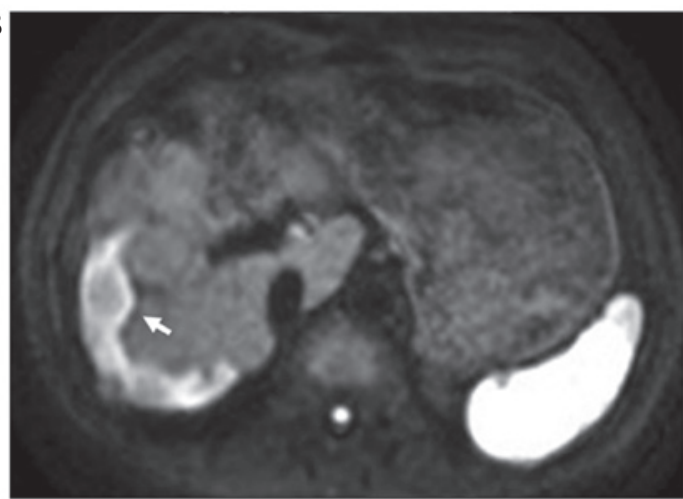

D

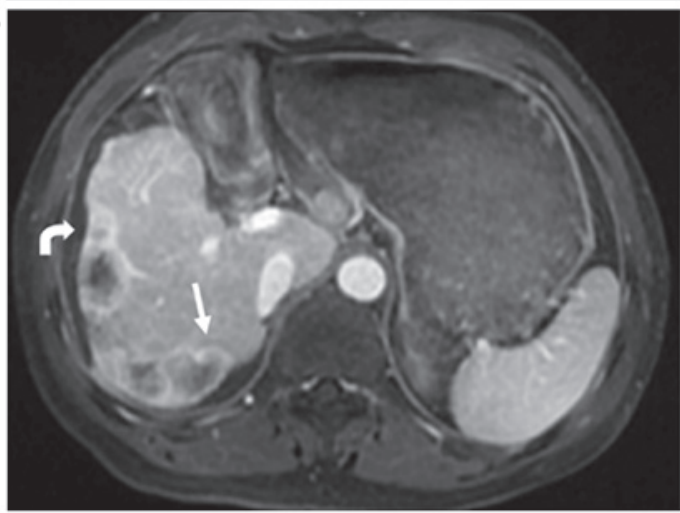

Figure 3. A 72-year-old male with multifocal nodular-type HEH. (A) On T2-weighted imaging, the coalescent lesions, locating in submarginal areas, showed 'white target-like' and 'strip-like' signs (arrow); the two-layered 'target' appearance was formed with a high-signal intensity core and peripheral slightly hyperintense halo. (B) On diffusion-weighted imaging, the lesions showed a high signal intensity halo outside of the central slightly high-signal intensity core (arrow). (C and D) On dynamic contrast-enhanced TI-weighted images with fat-suppression, HEH lesions showed gradual peripheral ring-like enhancement patterns with central low signal intensity in the arterial to delay phases; frequently the enhanced lesions were surrounded by a thin, hypointense ring in the portal or delay phases ('black target-like' sign) (arrow). Peripheral distribution and capsular retraction was also observed (curved arrow). HEH, hepatic epithelioid hemangioendothelioma. 
A



C

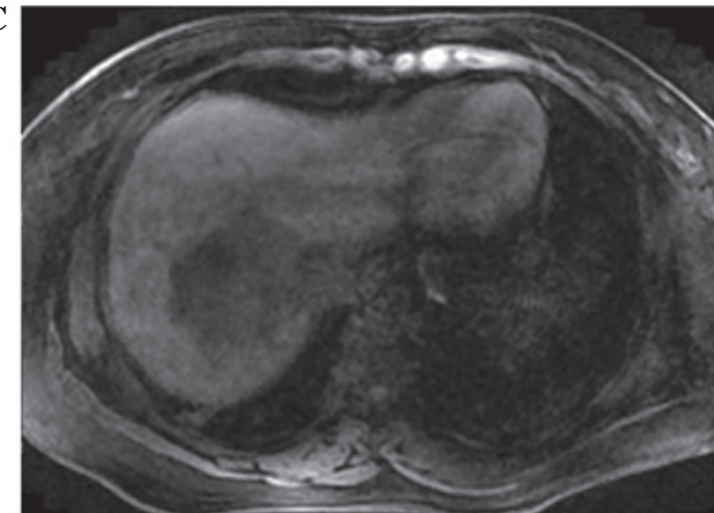

E



B

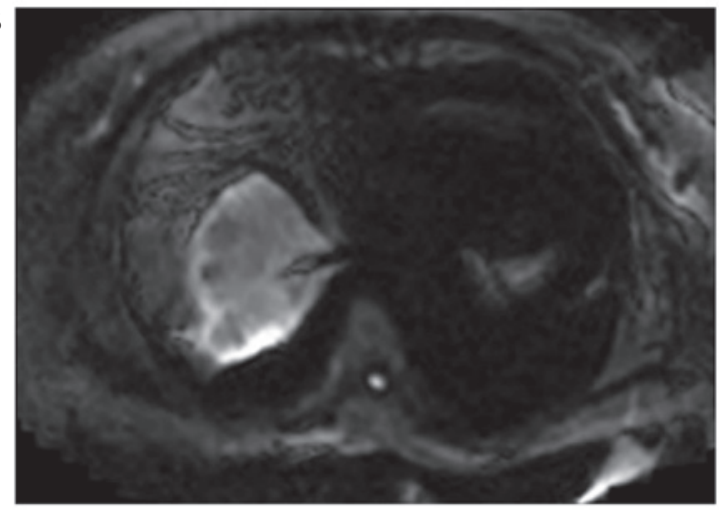

D

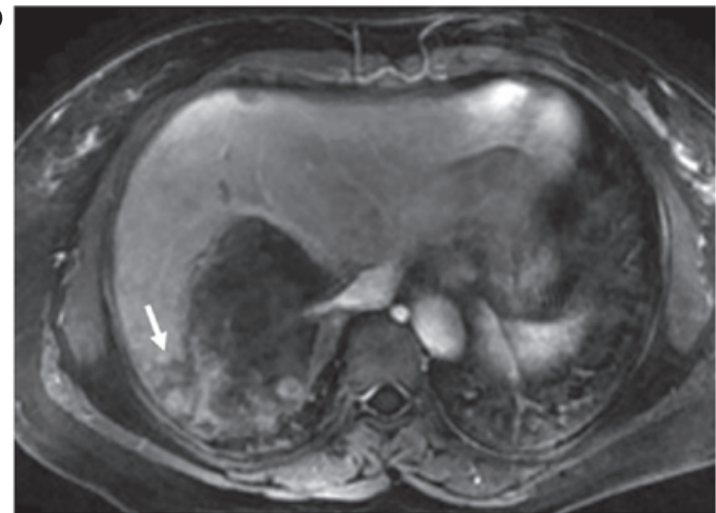

F

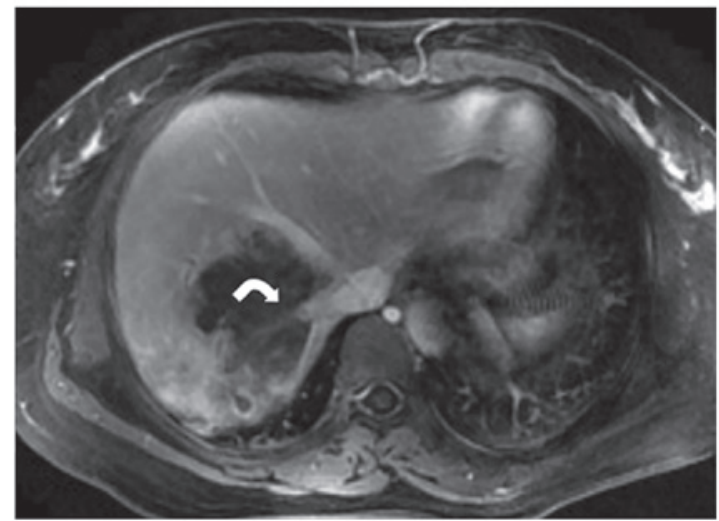

Figure 4. A 47-year-old male with multifocal nodular-type HEH. (A) Heterogeneous high-signal intensity masses were located in the right lobe surrounded by several nodules on T2-weighted imaging (arrow). (B) The lesions showed slightly high intensity with a high-intensity rim on diffusion-weighted imaging. (C) The lesions showed homogeneous low signal intensity on non-contrast TI weighted-imaging. (D) The lesions showed peripheral nodular enhancement in the arterial phase and gradual heterogeneous enhancement in the portal venous and delay phases (arrow). (E) The transient abnormal perfusion in peripheral liver parenchyma was visualized in arterial phase and disappeared in portal venous phase (black arrow). (F) A hepatic vein was found within the lesions ('lollipop' sign) (curved arrow). HEH, hepatic epithelioid hemangioendothelioma.

nodular lesions may be an earlier form of $\mathrm{HEH}$, as they later gradually transform into the diffuse type $(3,19,20)$.

In the current study, $\mathrm{HEH}$ was classified into three types according to the number of lesions: Solitary nodule, multifocal nodule and diffuse types. The percentage of solitary nodular-type cases $(7 \%)$ was markedly lower than that in previous reports $(2,4)$. This may be due to the fact that the imaging features of the solitary nodular-type disease are relatively non-specific (21).

On the contrary, multifocal nodular and diffuse types had a more typical appearance on CT and MRI. On non-contrast CT scans, tumors appeared as low density lesions with clear margins. On T1WI, the lesions exhibited low signal intensity whilst, on non-contrast T2-weighted fat-suppressed imaging, the lesions exhibited heterogeneously high signal intensity relative to the adjacent normal liver parenchyma. On DWI, the lesions appeared with slightly high-signal intensity cores and a high-signal intensity halo; these two-layered 'target-like' configurations were detected in 141 lesions (60.5\%).

On contrast-enhanced MRI, the enhancement features varied with different blood supply patterns. Multifocal lesions were classified into four categories according to different enhancement patterns: (i) Slightly irregular homogeneous enhancement; (ii) peripheral enhancement with central low signal intensity in the arterial phase, and enhanced lesions surrounded by a thin hypointense ring in the portal venous and delay phases ('black target-like' sign); (iii) nodular enhancement in the central part of the lesion in the arterial phase surrounded by ring-like enhancement in the portal venous and delay phases ('white target-like' sign); and 

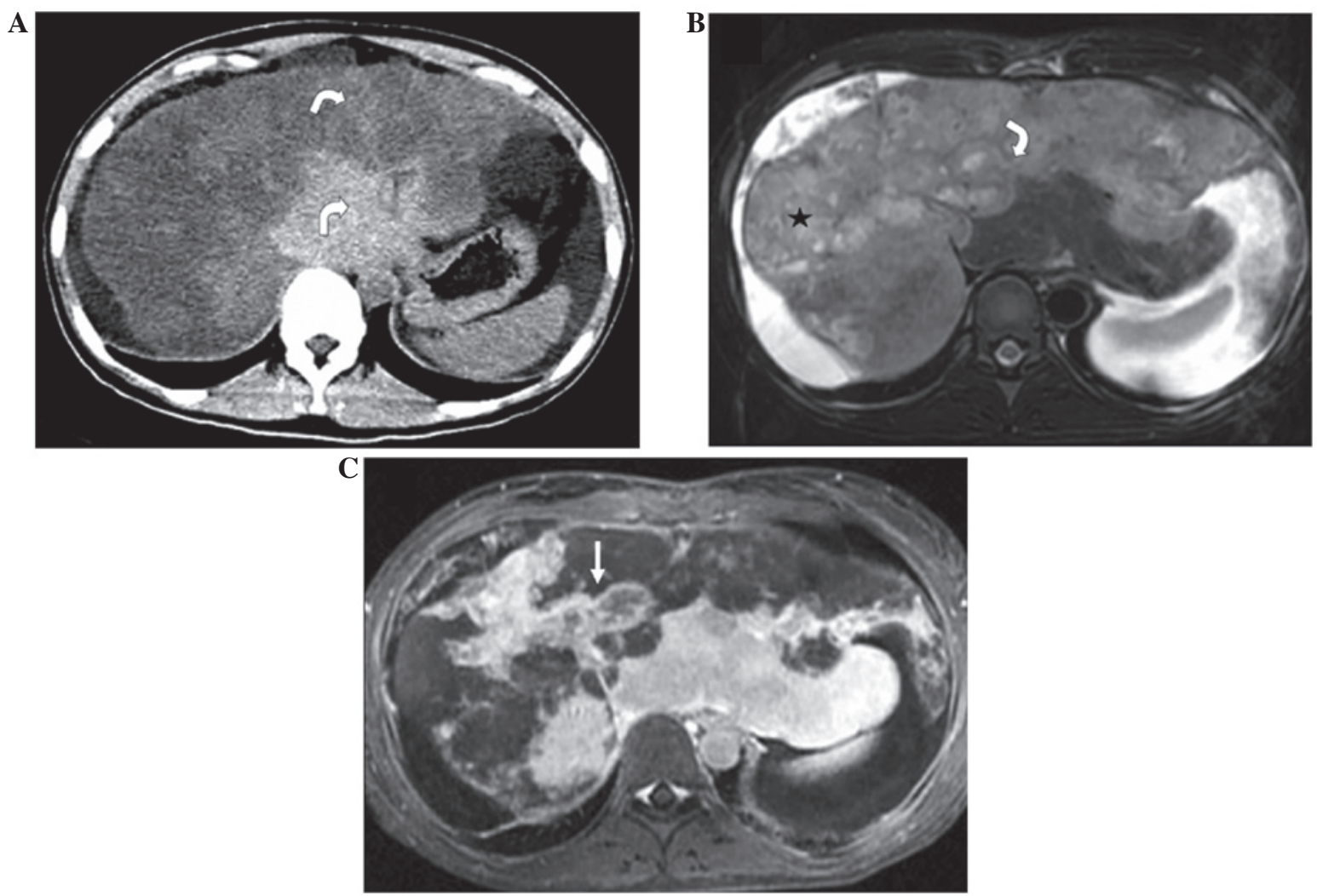

Figure 5. A 65-year-old female with diffuse-type HEH. (A) The CT features included diffuse low density lesions with minimal residual parts of normal liver parenchyma (curved arrow). (B and C) The MRI features included heterogeneous high signal intensity on T2-weighted imaging with patchy uniform signal intensity inside the lesions. (B) Nodules and masses (star) coexisted in this case. Nodules showed a 'target-like' sign (curved arrow) and heterogeneous enhancement on contrast-enhanced scan. (C) Masses exhibited 'strip-like' enhancement along central vessels in the arterial phase, and gradual enhancement in the portal venous and delay phases (straight arrow).

(iv) peripheral nodular enhancement in the arterial phase and centripetal enhancement in the portal venous and delay phases, an enhancement pattern that is more commonly indicated in hemangioma.

In the current study, $79 \%$ of lesions exhibited a 'target-like' sign on T2WI, and $70 \%$ of lesions exhibited the 'black target-like' sign on contrast-enhancement scans. This was consistent with previous reports $(9,22-25)$. In the study by Fan et al (23), the HEH was revealed to possess slightly increased signal intensity with increased signal intensity centers on the T2-weighted image, and 'ring-like' peripheral enhancement on the post-contrast enhanced MRI. In the study by Chen et al (9), HEH presented as two types (the multifocal and diffuse types), and $96.4 \%$ of the two types presented as the 'target sign' with a progressive enhancement rim on the contrast-enhanced multiple-phase MRI. The study by Bruegel et al (24) indicated that HEH showed a 'target-like' sign on T2, DWI and ADC maps, and the lesions also showed a variable degree of peripheral rim enhancement Notably, the 'white target-like' sign on contrast-enhanced imaging had not previously been described; we hypothesize that this unique sign may have value with regard to the diagnosis of $\mathrm{HEH}$. As the majority of cases were confirmed by needle biopsy, the relationship between the 'white target-like' sign and histopathological features remains unknown. As the core of the lesion had high signal intensity on T2WI and low signal intensity on contrast-enhanced T1WI, the 'white target-like' sign may correlate with central necrosis in the lesion. A thin, hypointense ring outside of a peripherally enhanced halo ('black target-like' sign) in the portal venous or delay phases may correlate to a layer of fibrous tissue between the lesion and the normal liver parenchyma (3). Certain previous studies have revealed that, during the hepatobiliary phase, the lesions may exhibit a contrast-enhanced core surrounded by a low-signal intensity halo; the central enhancement was indicated to be due to 'entrapment' of contrast agent in the central fibrous stroma, and the peripheral hypointense halo due to the lack of hepatobiliary enhancement in the peripheral tumor zone $(17,21)$.

In the present study, a 'lollipop' sign was also observed, as reported by Alomari (12). The detection of the involvement of the portal and hepatic veins on the CT and MRI scans were satisfactorily consistent with the pathology, as the scans were able to detect all involved portal and hepatic veins that were proven by pathological methods. In a study by Makhlouf et al (6), calcification was detected in $20 \%$ of lesions; however, none was observed in the current study, probably due to the insensitivity to calcification of MRI $(3,26,27)$. With regard to diffuse-type $\mathrm{HEH}$, similar CT features to the present study were reported by Baron et al (28).

In the current study, one case was misdiagnosed as hepatic fibrosis with a sub-marginal 'strip-like' sign; this sign may indicate the coalescence of multifocal nodules. None of the 14 cases in the study were diagnosed correctly based on CT and MRI findings, as the imaging characteristics of $\mathrm{HEH}$ were not widely recognized at the time of diagnosis, particularly for the solitary nodular-type cases. It is 
difficult to distinguish solitary HEH from metastatic tumors or cholangiocellular carcinoma (6).

The clinical symptoms of HEH vary greatly, and the disease may be asymptomatic or present with non-specific symptoms. Typically, HEH is present for a long time before a definitive diagnosis is made (2). For example, in the present study, hepatic lesions were found in 1 patient $\sim 4$ years prior to a definitive diagnosis. In the present study, 7 cases $(50 \%)$ had non-specific complaints, 3 cases (21\%) had right upper quadrant pain, 3 cases $(28.5 \%)$ had weight loss, 1 case $(7 \%)$ had jaundice attributable to the tumors, 1 case $(7 \%)$ had fever, and 1 case $(7 \%)$ had nausea. This was consistent with previous reports $(1,6)$. Makhlouf et al (6) collected 137 cases of HEH with nonspecific symptoms, including right upper quadrant pain or weight loss. In the study by Ishak et al (1), $12.5 \%$ of cases were asymptomatic. With regard to biochemical examinations, the concentrations of serum bilirubin, alkaline phosphatase and aspartate aminotransferase are typically significantly increased (2). In addition, the majority of cases are negative results for presence of tumor markers, and only a few cases exhibit slightly increased levels of CEA (26). In the present study, CEA levels were marginally increased in 1 case $(6.14 \mu \mathrm{g} / \mathrm{l}), \mathrm{CA} 125$ levels were increased in 1 case $(63 \mathrm{U} / \mathrm{ml})$, and tumor markers were negative in the other cases. Thus, the tumor markers most commonly used in clinical practice have no significant value for the diagnosis of $\mathrm{HEH}$.

Histologically, HEH is composed of dendritic and epithelioid cells that often contain vacuoles representing intracellular lumina. Furthermore, at least one of the endothelial markers (factor VIII-related antigen, CD34 and/or CD31) is positively expressed (6,29). Microscopically, characteristic spindle- and oval-shaped cells with abundant eosinophilic cytoplasm are observed (30). Commonly, biopsy is able to confirm a diagnosis of $\mathrm{HEH}$; however, an insufficient size of biopsy specimen may results in failure to diagnose to $\mathrm{HEH}$, and laparoscopic liver biopsy is preferred in order to obtain adequate specimens for analysis (31). Fortunately, in the current study, definitive diagnosis could be made with needle biopsy in the majority of cases.

Management strategies for $\mathrm{HEH}$ include liver resection, liver transplantation, transcatheter arterial chemoembolization and palliative treatment $(2,32)$. Recent studies have suggested that immediate treatment may not be the optimal strategy (33). $\mathrm{HEH}$ often follows an indolent course. In a previous study, there was no difference of 5-year survival in patients with unilateral or bilateral lesions, localized or metastatic disease, even with an initial treatment regimen of surgery (33) Therefore, the biological behavior of the tumor may be associated, in part, with its matrix, which may exhibit inflammation, dense sclerosis and calcification. Assessment of disease behavior may better stratify treatment options (33). For unresectable HEH, liver transplantation is the best option; studies have demonstrated that the long-term survival time is very good, and much better than in HCC patients (29). One study indicated that $62.5 \%$ of patients with HEH survived for over 5 years, and one patient succumbed 28 years subsequent to the initial diagnosis (1).

The limitations of the present study include its retrospective nature, the fact that it was conducted in a single center, and the small sample size. Further studies are required to identify more novel signs associated with HEH.
In conclusion, $\mathrm{HEH}$ is a rare tumor that may present certain typical imaging features, including the 'white target-like', 'black target-like', 'lollipop' and 'strip-like' signs, capsular contraction, submarginal distribution. These features may contribute significantly to the definitive diagnosis of $\mathrm{HEH}$. Use of a variety of MRI sequences may provide more information for the differential diagnosis of $\mathrm{HEH}$.

\section{References}

1. Ishak KG, Sesterhenn IA, Goodman ZD, Rabin L and Stromeyer FW: Epithelioid hemangioendothelioma of the liver: A clinicopathologic and follow-up study of 32 cases. Hum Pathol 15: 839-852, 1984.

2. Mehrabi A, Kashfi A, Fonouni H, Schemmer P, Schmied BM Hallscheidt P, Schirmacher P, Weitz J, Friess H, Buchler MW and Schmidt J: Primary malignant hepatic epithelioid hemangioendothelioma: A comprehensive review of the literature with emphasis on the surgical therapy. Cancer 107: 2108-2121, 2006.

3. Miller WJ, Dodd GD III, Federle MP and Baron RL: Epithelioid hemangioendothelioma of the liver: Imaging findings with pathologic correlation. AJR Am J Roentgenol 159: 53-57, 1992.

4. Salech F, Valderrama S, Nervi B, Rodriguez JC, Oksenberg D, Koch A, Smok G, Duarte I, PerezAyuso RM, Jarufe N, Martinez J, Soza A, Arrese M and Riquelme A. Thalidomide for the treatment of metastatic hepatic epithelioid hemangioendothelioma: a case report with a long term follow-up. Ann Hepatol 2011; 10: 99-102

5. Sangro B, Inarrairaegui M and Fernandez-Ros N. Malignant epithelioid hemangioendothelioma of the liver successfully treated with Sorafenib. Rare Tumors 2012; 4: e34

6. Makhlouf HR, Ishak KG and Goodman ZD: Epithelioid hemangioendothelioma of the liver: A clinicopathologic study of 137 cases. Cancer 85: 562-582, 1999.

7. Neofytou K, Chrysochos A, Charalambous N, Dietis M, Petridis C, Andreou C and Petrou A: Hepatic epithelioid hemangioendothelioma and the danger of misdiagnosis: Report of a case. Case Rep Oncol Med 2013: 243939, 2013

8. Azzam RI, Alshak NS and Pham HP: AIRP best cases in radiologic-pathologic correlation: Hepatic epithelioid hemangioendothelioma. Radiographics 32: 789-794, 2012.

9. Chen Y, Yu RS, Qiu LL, Jiang DY, Tan YB and Fu YB: Contrast-enhanced multiple-phase imaging features in hepatic epithelioid hemangioendothelioma. World J Gastroenterol 17: 3544-3553, 2011

10. Hamilton SR and Aaltonen LA (eds): Tumours of the Liver and Intrahepatic Bile Ducts. In: World Health Organization Classification of Tumours. Pathology and Genetics of Tumours of the Digestive System. IARC Press, Lyon, p158, 2000.

11. Klotz T, Montoriol PF, Da Ines D, Petitcolin V, Joubert-Zakeyh J and Garcier JM: Hepatic haemangioma: Common and uncommon imaging features. Diagn Interv Imaging 94: 849-859, 2013.

12. Alomari AI: The lollipop sign: A new cross-sectional sign of hepatic epithelioid hemangioendothelioma. Eur J Radiol 59: 460-464, 2006.

13. Okano H, Nakajima H, Tochio T, Suga D, Kumazawa H, Isono Y, Tanaka H, Matsusaki S, Sase T, Saito T, et al: A case of a resectable single hepatic epithelioid hemangioendothelioma with characteristic imaging by ADC map. Clin J Gastroenterol 8: 406-413, 2015.

14. Weiss SW and Enzinger FM: Epithelioid hemangioendothelioma: A vascular tumor often mistaken for a carcinoma. Cancer 50: 970-981, 1982.

15. Verbeken E, Beyls J, Moerman P, Knockaert D, Goddeeris P and Lauweryns JM: Lung metastasis of malignant epithelioid hemangioendothelioma mimicking a primary intravascular bronchioalveolar tumor. A histologic, ultrastructural and immunohistochemical study. Cancer 55: 1741-1746, 1985.

16. Idilman R, Dokmeci A, Beyler AR, Bastemir M, Ormeci N, Aras N, Ekinci C, Uzunalimoglu O, De Maria N and Van Thiel DH: Successful medical treatment of an epithelioid hemangioendothelioma of liver. Oncology 54: 171-175, 1997.

17. Paolantonio P, Laghi A, Vanzulli A, Grazioli L, Morana G, Ragozzino A and Colagrande S: MRI of hepatic epithelioid hemangioendothelioma (HEH). J Magn Reson Imaging 40: $552-558,2014$. 
18. Zhao XY, Rakhda MI, Habib S, Bihi A, Muhammad A, Wang TL and Jia JD: Hepatic epithelioid hemangioendothelioma: A comparison of Western and Chinese methods with respect to diagnosis, treatment and outcome. Oncol Let 7: 977-983, 2014.

19. Furui S, Itai Y, Ohtomo K, Yamauchi T, Takenaka E, Iio M, Ibukuro K, Shichijo Y and Inoue Y: Hepatic epithelioid hemangioendothelioma: Report of five cases. Radiology 171: 63-68, 1989.

20. Van Beers B, Roche A, Mathieu D, Menu Y, Delos M, Otte JB, Lalonde L and Pringot J: Epithelioid hemangioendothelioma of the liver: MR and CT findings. J Comput Assist Tomogr 16: 420-424, 1992.

21. Kim EH, Rha SE, Lee YJ, Yoo IR, Jung ES and Byun JY: CT and MR imaging findings of hepatic epithelioid hemangioendotheliomas: Emphasis on single nodular type. Abdom Imaging 40: 500-509, 2015

22. Lin J and Ji Y: CT and MRI diagnosis of hepatic epithelioid hemangioendothelioma. Hepatobiliary Pancreat Dis Int 9: 154-158, 2010.

23. Fan F, Yang X, Zhu B and Zhang Y: Clinical and radiological characteristics of Chinese patients with hepatic epithelioid hemangioendothelioma. Ann Saudi Med 33: 334-338, 2013.

24. Bruegel M, Muenzel D, Waldt S, Specht K and Rummeny EJ: Hepatic epithelioid hemangioendothelioma: Findings at CT and MRI including preliminary observations at diffusion-weighted echo-planar imaging. Abdom Imaging 36: 415-424, 2011.

25. Lyburn ID, Torreggiani WC, Harris AC, Zwirewich CV Buckley AR, Davis JE, Chung SW, Scudamore CH and Ho SG: Hepatic epithelioid hemangioendothelioma: Sonographic, CT and MR imaging appearances. AJR Am J Roentgenol 180: 1359-1364, 2003.
26. Earnest Ft IV and Johnson CD: Case 96: Hepatic epithelioid hemangioendothelioma. Radiology 240: 295-298, 2006.

27. Bartolozzi C, Cioni D, Donati F and Lencioni R: Focal liver lesions: MR imaging-pathologic correlation. Eur Radiol 11: 1374-1388, 2001

28. Baron PW, Amankonah T, Cubas RF, Kore AH, Elihu A, de Vera ME and Perez MC: Diffuse hepatic epithelioid hemangioendothelioma developed in a patient with hepatitis $\mathrm{C}$ cirrhosis. Case Rep Transplant 2014: 694903, 2014.

29. Remiszewski P, Szczerba E, Kalinowski P, Gierej B, Dudek K, Grodzicki M, Kotulski M, Paluszkiewicz R, Patkowski W, Zieniewicz K and Krawczyk M: Epithelioid hemangioendothelioma of the liver as a rare indication for liver transplantation. World J Gastroenterol 20: 11333-11339, 2014.

30. Kubota S, Baba H, Kumamoto K, Hatano S, Amano K Ohsawa T, Okada T, Kumagai Y, Ishibashi K, Haga N, et al: A case of multiple hepatic epithelioid hemangioendothelioma mimicking metastatic hepatic tumor. Gan To Kagaku Ryoho 39: 2012-2014, 2012 (In Japanese).

31. Deng Y, Zhou Y and Cheng N: Laparoscopic liver biopsy in the diagnosis of hepatic epithelioid hemangioendothelioma: A case report. Oncol Let 8: 1317-1319, 2014.

32. Mistry AM, Gorden DL, Busler JF, Coogan AC and Kelly BS: Diagnostic and therapeutic challenges in hepatic epithelioid hemangioendothelioma. J Gastrointest Cancer 43: 521-525, 2012.

33. Thomas RM, Aloia TA, Truty MJ, Tseng WH, Choi EA, Curley SA, Vauthey JN and Abdalla EK: Treatment sequencing strategy for hepatic epithelioid haemangioendothelioma. HPB (Oxford) 16: 677-685, 2014. 